\title{
La esclavitud como indicador del desempeño económico neogranadino en el siglo XVII
}

\author{
Carlos Eduardo Valencia Villa \\ Universidad Nacional de Colombia
}

\begin{abstract}
Resumen
Este artículo analiza la correlación existente entre la fuerza de trabajo esclava y el desempeño económico de la Nueva Granada durante el siglo XVII, proponiendo un método para estudiar el comportamiento general de la economía colonial. El modelo de interpretación se sustenta en tres elementos principales. Se propone una expresión analítica alternativa del PIB, más simple de la usada comúnmente por los economistas; se hace una relación entre la economía y el número total de esclavos, como primera variable de correlación y, por último, se expone la vinculación entre el comportamiento de los precios de los esclavos y el desempeño económico general.
\end{abstract}

\begin{abstract}
Este artículo analiza la correlación existente entre la fuerza de trabajo esclava y el desempeño económico de la Nueva Granada durante el siglo XVII, proponiendo un método para estudiar el comportamiento general de la economía colonial. El modelo de interpretación se sustenta en tres elementos principales. Se propone una expresión analítica alternativa del PIB, más simple de la usada comúnmente por los economistas; se hace una relación entre la economía y el número total de esclavos, como primera variable de correlación y, por último, se expone la vinculación entre el comportamiento de los precios de los esclavos y el desempeño económico general.
\end{abstract}




\section{Presentación}

Es evidente el papel fundamental de la esclavitud en la generación de la riqueza económica de las colonias americanas durante los siglos XVI, XVII y XVIII. En especial en el caso neogranadino, su actuación ha sido resaltada por todos aquellos que la han investigado. ${ }^{1}$ Por eso este artículo no está pensado para discutir la participación esclava en la vida económica, ni mucho menos para demostrar este rol fundamental. Lo que se pretende aquí es indagar acerca de la correlación existente entre la fuerza de trabajo esclava y el desempeño económico, esto es, intentar precisar si las variables que representan la historia económica tienen un correlato en el precio y volumen de los esclavos que se transaban, de tal manera que sea posible comprobar que los auges y caídas de la economía, como un todo, son verificables en el universo esclavo.

Así, no se va a proponer que la fuerza de trabajo esclava determina el devenir de la economía de la Nueva Granada, hipótesis que sería completamente reduccionista. Lo que se busca es proponer el esquema de un método para indicar el comportamiento general de la economía. Este consiste en mostrar que los ciclos del mercado de esclavos se vinculan de manera directamente proporcional con los ciclos de la producción, con lo cual se pueda inferir que expansiones o contracciones del mercado de esclavos son seguidas de fenómenos similares en el sistema en general. Por eso, entonces, no vamos a proponer que el sistema económico se explique a través de la esclavitud. Lo único en lo que pretendemos indagar es acerca de la relación entre economía en general y esclavitud en particular, de tal manera que podamos establecer los ciclos del mercado de esclavos como indicadores, y el término indicador debe ser comprendido en su acepción más simple, es decir, no como una forma de explicación, sino como un mecanismo de representar la realidad. Por lo tanto, la aspiración que tenemos aquí es la de demostrar que las tendencias de la economía y de la esclavitud se comportan de manera paralela, por lo cual si es posible construir una serie para una, entonces se pueda inferir, de manera legítima, que la otra presenta un comportamiento similar. Lo cual no implica que estemos afirmando que la segunda serie se reduzca a la primera.

\footnotetext{
1 Jaime Jaramillo Uribe, "Esclavos y señores en la sociedad colombiana del siglo XVIII," Anuario Colombiano de Historia Social y de la Cultura 1, no. 1 (1963); Anthony McFarlane, Colombia antes de la Independencia: Economía, sociedad y política bajo el dominio borbón (Bogotá: Banco de la República, 1997); y Germán Colmenares, Historia económica y social de Colombia: Popayán una sociedad esclavista, 1680-1800 (Bogotá: La Carreta, 1979).
} 
Para sustentar el modelo de interpretación que se pretende exponer, se van a seguir tres pistas. La primera se refiere a un pequeño recuento de las posiciones conceptuales que se han formulado para comprender la generación del valor económico, de tal forma que se llegue a una expresión analítica alternativa del PIB, la cual debe ser más simple que la que comúnmente utilizan los economistas. Esto con el fin de poder dar cuenta de las particularidades de la economía colonial neogranadina, a la vez que permitirá simplificar algunos de los argumentos que se pretenden dar. El segundo momento de la exposición, pasará a través de la relación de la economía con el total de esclavos que trabajan en Nueva Granada, como primera variable de correlación. Por último, se expondrá la vinculación entre el comportamiento de los precios de los esclavos y el de la economía.

Antes de comenzar, es importante aclarar que la propuesta que se presenta está limitada al caso de Nueva Granada en el siglo XVII y no es posible generalizarla más allá, en tanto en varias ocasiones, como el lector fácilmente percibirá, se acude a fenómenos de cuya existencia sólo se han verificado para esa región y en ese momento particular.

\section{Generación de valor}

En el establecimiento del PIB de una economía lo que generalmente se hace es sumar todos los valores agregados que producen cada uno de los sectores que la conforman. Sin embargo, este procedimiento es poco útil a la hora de intentar comprender el comportamiento económico de una sociedad donde los registros pormenorizados de todas las actividades no se han llevado. Por lo tanto, es preferible volver a la definición básica de Producto Interno Bruto y desde ella buscar reconstruir la formula. Según esta definición, el PIB es la sumatoria de todos los valores agregados a precio de los factores, sin descontar la depreciación. Lo cual quiere decir que la sumatoria por sectores es simplemente una forma de ordenar de manera sistemática la expresión, pero lo que realmente importa es lograr establecer los valores que se han generado y luego totalizarlos para la economía en general.

De esa manera, lo que es evidente es que lo importante es dirigir la mirada hacia la generación de valor, y para los intereses de este artículo se pueden obviar los sectores particulares a que ha estado vinculado el crecimiento o decrecimiento económico.

Ahora bien, las teorías acerca del valor han hecho énfasis en alguno de los tres factores siguientes, o en combinaciones de estos: recursos naturales (especialmente la tierra, pero también incluyendo algunos otros como las minas, los lagos y mares), el trabajo 
humano, o en los bienes de capital. Por lo cual, el PIB podría ser reformulado como la suma del valor generado por los recursos naturales, el trabajo y el capital. Cada uno de los cuales estaría multiplicado por un índice determinado que daría cuenta del peso específico de estas formas dentro de la sociedad particular que se este observando. En otras palabras, el PIB se podría reescribir como:

$$
\mathrm{PIB}=\eta \mathrm{R}+\phi \mathrm{T}+\gamma \mathrm{K}
$$

O de manera más general,

$$
\mathrm{PIB}=f(\mathrm{R}, \mathrm{T}, \mathrm{K})
$$

En la cual R, T y $\mathrm{K}$ representan a los valores agregados por los recursos naturales, el trabajo y el capital respectivamente. A su vez, $\eta, \phi$ y $\gamma$ son los índices particulares que dan cuenta de la contribución de cada una de las formas al total general.

Esta expresión sería poco útil si se deseara establecer con claridad cuánto es el PIB de una economía o el porcentaje en que ella ha crecido, sin embargo, para los fines que aquí se tienen es pertinente, en tanto sitúa el problema en términos de los factores de producción de valor y no de los sectores que lo producen, pasando así de variables concretas y particulares y desafortunadamente desconocidas, a otras generales y por ende ubicables en el plano de la mera abstracción, es decir, deducibles matemáticamente.

El siguiente paso es ver de manera particular cada una de las formas de producción de valor que componen el PIB, dándoles una mirada de función matemática, esto es, buscando comprender de cuáles variables depende su desempeño.

\section{A. Recursos naturales}

Los recursos naturales participan en la generación de valor, en tanto de ellos provienen todas las materias primas de los procesos manufacturados. Sobre esto se han escrito montones de páginas y no es la pretensión de este artículo debatir sobre los alcances de las posiciones conceptuales que han hecho hincapié en este factor. Lo importante aquí es resaltar que en la economía colonial este papel es de vital importancia cuando se trata de 
los recursos mineros, ${ }^{2}$ aunque en alguna medida también de los agrícolas, pesqueros y ganaderos.

Los recursos naturales, en cuanto a la generación de valor se trata, son completamente pasivos, es decir, no depende exclusivamente de ellos la producción. Simplemente son los que abren o restringen las posibilidades de su manufactura. Por ejemplo, el acceso a los metales en una mina es una variable fundamental para el cálculo de la rentabilidad de ésta. Sin embargo, del mero hecho de la facilidad para extraerlos no se puede deducir que hayan sido extraídos. Esto dependerá de la fuerza de trabajo de que se disponga. ${ }^{3}$ Aunque, si se tiene fuerza de trabajo, pero el acceso es imposible, de acuerdo a la técnica del momento, entonces, tampoco se podrá realizar explotación alguna.

Así, se puede afirmar que los recursos naturales son una condición necesaria en la producción de valor, pero no una condición suficiente, en tanto su papel es meramente pasivo (no por ello despreciable) y dependerá del acceso a la fuerza de trabajo. Conclusión a la que han llegado muchos trabajos de la economía colonial en general.

Esto permite reescribir el valor agregado por los recursos naturales de la siguiente forma:

$$
\mathrm{R}=\lambda \mathrm{T}
$$

Donde $\lambda$ es un factor que expresa el acceso a los recursos naturales y $\mathrm{T}$ es el valor agregado del trabajo. Lo cual supone que si el $\lambda$ es cero, es porque el acceso es francamente imposible y esta forma de valor no se hace presente. Mientras que si el $\lambda$ crece, entonces el $\mathrm{R}$ crece de manera proporcional, lo cual a su vez deberá producir incrementos en T, para crear un efecto multiplicador sobre toda la economía. Así, en términos de funciones matemáticas, se escribiría como $\mathrm{R}=f(\lambda, \mathrm{T})$

\section{B. Fuerza de trabajo}

El énfasis en la fuerza de trabajo como generadora de valor ha sido discutida, al menos, desde los tiempos de David Ricardo y Karl Marx y por ende basta con recordar que la

\footnotetext{
2 Jacob Gorender, "La América portuguesa y el esclavismo colonial," en Los conquistados y la población indígena de las Américas, comp. Heraclio Bonilla (Bogotá: Tercer Mundo, 1992).

${ }^{3}$ Julián B. Ruiz Rivera, La plata de Mariquita en el siglo XVII: Mita y producción, Cuadernos de historia, no. 5 (Tunja: Nuestra América, 1979).
} 
fuerza de trabajo particular es la que produce los valores de uso de las mercancías; y el trabajo general, abstracto e igual, es el que se representa en los valores de cambio, con lo cual, las mercancías son equiparables en cuanto a la cantidad de trabajo contenidas en ellas. $^{4}$

Como es bien conocido, en el caso de la Nueva Granada existieron varios tipos de trabajo, vinculados a formas disímiles de coacción de los grupos que los ofrecían. Estos eran: indígenas, esclavos y libres, quienes respondían a formas diferentes de explotación y por ende se enfocaban a sectores diferentes de la economía, lo cual pone restricciones acerca de las posibilidades de sustitución de un tipo de mano de obra por otra. Sin embargo, esta restricción es relevante para el análisis de un individuo o sector económico en particular. Pero como los objetivos de este artículo se dirigen a la economía en su conjunto y se ha hecho énfasis no en la composición sectorial, sino en las fuentes del valor, entonces la mercancía trabajo es sustituible para la economía como totalidad, en cada una de sus formas particulares, tal como lo sería cualquier otro bien o servicio, esto es, dependiendo de su precio y de la utilidad que produzca.

De esta manera, la composición de la fuerza de trabajo empleada en la Nueva Granada es tanto una función del precio de cada una de las formas de trabajo, como del acceso y utilidad derivadas de ellas. La cuestión del precio se tratará un poco más adelante, por ahora se concentrará la atención en el problema de la cantidad de fuerza de trabajo disponible, con lo cual, el valor generado por el trabajo se puede reescribir como:

$$
\mathrm{T}=\mathrm{T}_{\mathrm{e}}+\mathrm{T}_{\mathrm{o}}
$$

$T_{e}$ representa el valor agregado por el trabajo esclavo y $T_{o}$ es el valor generado por las otras formas de trabajo. Ahora bien, como las dos formas son sustituibles, entonces entre ellas existe una relación que puede ser pensada por lo que en microeconomía se conoce como una curva de indiferencia, es decir, que para mantener la utilidad derivada de ellas constante, se pueden establecer parejas de $\mathrm{T}_{\mathrm{e}} \mathrm{y} \mathrm{T}_{0}$. De tal manera que si se eleva el empleo de una de las formas, se supone que la otra se reduce, y así se mantiene la utilidad invariable. A su vez, para elevar la utilidad, entonces las dos fuentes de valor vía trabajo se elevan dando como resultado una nueva curva de indiferencia, en la que aparecerán otras combinaciones de parejas. Así, $\mathrm{T}_{\mathrm{e}} \mathrm{y} \mathrm{T}_{\mathrm{o}}$ se autodeterminan, esto es, que

\footnotetext{
${ }^{4}$ Karl Marx, Contribución a la crítica de la economía política (Madrid: Siglo XXI, 1997).
} 


$$
\mathrm{T}_{\mathrm{o}}=g\left(\mathrm{~T}_{\mathrm{e}}\right)
$$

En otras palabras, la cantidad de valor agregado por el trabajo no esclavo es una función que depende del trabajo agregado por los esclavos (aunque esta relación podría escribirse también al revés y hacer depender la fuerza de trabajo esclava de la no esclava). Dependencia que es inversamente proporcional.

Así la función compuesta que expresa el valor agregado por la fuerza de trabajo es:

$$
\mathrm{T}=f\left(\mathrm{~T}_{\mathrm{e}}, \mathrm{T}_{\mathrm{o}}\right)=f\left(\mathrm{~T}_{\mathrm{e}}, g\left(\mathrm{~T}_{\mathrm{e}}\right)\right)
$$

\section{Bienes de capital}

Por otro lado y en la orilla opuesta a las posiciones que defienden el trabajo como fuente única de generación de valor en la economía, se encuentran las posiciones que hacen hincapié en el papel de los bienes de capital, entendidos estos como los activos que permiten producir otros bienes y con ello amplían la capacidad de crear valor en una sociedad. Ahora bien, estos bienes de capital se establecen a través de la ecuación:

$$
\mathrm{K}=\mathrm{S}-\mathrm{E}+\mathrm{M}
$$

Donde $\mathrm{K}$ representa la formación de capital, $\mathrm{S}$ el ahorro, E el volumen de las exportaciones y $\mathrm{M}$ el de las importaciones, todos los cuales se expresan en términos monetarios.

Como es evidente, el ahorro de una sociedad es la diferencia entre la producción y el consumo luego de descontados los impuestos, es decir, que S depende de la generación de valor y en el caso particular de la Nueva Granada esto quiere decir de la fuerza de trabajo. De manera similar sucede con las exportaciones del Nuevo Reino, en tanto su principal producto de exportación fueron los minerales. ${ }^{5}$ Por su parte, el grueso de las importaciones se concentraba en dos rubros, el tráfico negrero por Cartagena ${ }^{6}$ y los situados fiscales. Pero los segundos no presentan un comportamiento homogéneo en el

\footnotetext{
${ }^{5}$ Germán Colmenares, Historia económica y social de Colombia I, 1537-1719 (1973; Bogotá: Tercer Mundo, 1997).

${ }^{6}$ Zacarías Moutoukias, "Contrabando y sector externo en Hispanoamérica colonial," en Los nudos 1, vol. 2 de Para una historia de América, coord. Marcello Carmagnani, Alicia Hernández Chávez y Ruggiero Romano (México, D.F.: Fondo de Cultura Económica, 1999).
} 
tiempo, en tanto estos giros se realizaban en coyunturas muy puntuales, vinculadas a esfuerzos bélicos, es decir, que los efectos de formación de capital y de los eslabonamientos que vienen con ellos tenían una dimensión muy limitada y focalizada.

Así, se puede reescribir la inversión en bienes de capital para este caso en particular como:

$$
\mathrm{K}=f\left(\mathrm{~T}, \mathrm{M}_{\mathrm{e}}\right)
$$

$\mathrm{T}$ de nuevo es el valor generado por el trabajo $\mathrm{y}_{\mathrm{e}}$ es el volumen de esclavos importados.

\section{Comportamiento y correlación: Volumen de esclavos}

Con todas las variables que crean valor en la economía expresadas como funciones matemáticas, es posible empezar a discutir la correlación entre el crecimiento o decrecimiento en general con el comportamiento de la fuerza de trabajo esclava. Se empezará con el caso del volumen de los esclavos, es decir, con la disposición de su fuerza de trabajo.

En las paginas anteriores se ha dicho que el PIB es una función de los recursos naturales, del trabajo y de los bienes de capital, es decir, $\mathrm{PIB}=f(\mathrm{R}, \mathrm{T}, \mathrm{K})$, que a su vez puede ser reescrita, si se expresa cada una de las variables como funciones:

$$
\mathrm{PIB}=f\left(\mathrm{R}(\lambda, \mathrm{T}), \mathrm{T}\left(\mathrm{T}_{\mathrm{e}}, \mathrm{T}_{\mathrm{o}}\right), \mathrm{K}\left(\mathrm{T}, \mathrm{M}_{\mathrm{e}}\right)\right.
$$

o más explícitamente

$$
\mathrm{PIB}=f\left(\mathrm{R}\left(\lambda, \mathrm{T}\left(\mathrm{T}_{\mathrm{e}}, \mathrm{T}_{\mathrm{o}}\right)\right), \mathrm{T}\left(\mathrm{T}_{\mathrm{e}}, \mathrm{T}_{\mathrm{o}}\right), \mathrm{K}\left(\mathrm{T}\left(\mathrm{T}_{\mathrm{e}}, \mathrm{T}_{\mathrm{o}}\right), \mathrm{M}_{\mathrm{e}}\right)\right)
$$

Pero como $\mathrm{T}_{\mathrm{o}}=g\left(\mathrm{~T}_{\mathrm{e}}\right)$, entonces la función del PIB sería:

$$
\mathrm{PIB}=f\left(\mathrm{R}\left(\lambda, \mathrm{T}\left(\mathrm{T}_{\mathrm{e}}, g\left(\mathrm{~T}_{\mathrm{e}}\right)\right)\right), \mathrm{T}\left(\mathrm{T}_{\mathrm{e}}, g\left(\mathrm{~T}_{\mathrm{e}}\right)\right), \mathrm{K}\left(\mathrm{T}\left(\mathrm{T}_{\mathrm{e}}, g\left(\mathrm{~T}_{\mathrm{e}}\right)\right), \mathrm{M}_{\mathrm{e}}\right)\right)
$$

La función PIB, es una función que en principio depende de tres variables, pero estas a su vez dependen de otras variables que finalmente están vinculadas a otras, esto es, que se tiene una función compuesta . 
Ahora bien, como lo que se pretende es observar el comportamiento del PIB, entonces se debe calcular el gradiente, método que se emplea para establecer los ritmos de crecimiento de una función matemática que tiene dos o más variables independientes. El gradiente es el vector de derivadas parciales, en los cuales cada componente del vector expresa una de las direcciones en las que se mueve la superficie total. Superficie entendida aquí como la representación geométrica de la función, pero que en este caso no sería posible trazar, pues se trata de una superficie en cuatro dimensiones.

En términos matemáticos se diría:

$$
\nabla \mathrm{PIB}=(\delta \mathrm{PIB} / \delta \lambda)^{\wedge} \lambda+\left(\delta \mathrm{PIB} / \delta \mathrm{T}_{\mathrm{e}}\right)^{\wedge} \mathrm{T}_{\mathrm{e}}+\left(\delta \mathrm{PIB} / \delta \mathrm{M}_{\mathrm{e}}\right)^{\wedge} \mathrm{M}_{\mathrm{e}}
$$

En el cual $\boldsymbol{\nabla}$ representa al operador gradiente $\mathrm{y}^{\wedge}$ indica la dirección de la derivada parcial y por ende la componente particular del vector.

Para calcular el gradiente sería necesario calcular cada una de las derivadas parciales, ejercicio que consiste en dejar constantes todas las variables menos aquella con respecto a la que se está derivando. Además, es importante aclarar que en algunos casos es necesario sacar la derivada interna, en tanto se tiene una función compuesta. En resumen, se tiene:

$(\delta \mathrm{PIB} / \delta \lambda)=1, \quad$ Porque la derivada de una constante es igual a cero y $\lambda$ es un operador lineal y por ende su derivada es 1. Estrictamente, podría ser positivo o negativo, en tanto el acceso a los recursos naturales creciera o decreciera, respectivamente.

$\left(\delta \mathrm{PIB} / \delta \mathrm{T}_{\mathrm{e}}\right)=\left[\left((\delta \mathrm{R} / \delta \mathrm{T})\left(\delta \mathrm{T} / \delta \mathrm{T}_{\mathrm{e}}\right)\right),\left((\delta \mathrm{T} / \delta \mathrm{g})\left(\delta \mathrm{g} / \delta \mathrm{T}_{\mathrm{e}}\right)\right)\right],\left[\left(\delta \mathrm{T} / \delta \mathrm{T}_{\mathrm{e}}\right),\left((\delta \mathrm{T} / \delta \mathrm{g})\left(\delta \mathrm{g} / \delta \mathrm{T}_{\mathrm{e}}\right)\right)\right]$, $\left[\left((\delta \mathrm{K} / \delta \mathrm{T})\left(\delta \mathrm{T} / \delta \mathrm{T}_{\mathrm{e}}\right)\right),\left((\delta \mathrm{K} / \delta \mathrm{T})(\delta \mathrm{T} / \delta \mathrm{g})\left(\delta \mathrm{g} / \delta \mathrm{T}_{\mathrm{e}}\right)\right)\right]$

$\left(\delta \mathrm{PIB} / \delta \mathrm{M}_{\mathrm{e}}\right)=\delta \mathrm{K} / \delta \mathrm{M}_{\mathrm{e}} \quad$ En tanto la importación de esclavos es una función que durante el siglo XVII representa volúmenes variables de 
introducción, aunque su comportamiento, en términos de tendencia, fue siempre decreciente. ${ }^{7}$

Para simplificar la notación, se dejara la segunda derivada parcial indicada, con lo que el gradiente es:

$$
\nabla \mathrm{PIB}=1^{\wedge} \lambda+\left(\delta \mathrm{PIB} / \delta \mathbf{T}_{\mathrm{e}}\right)^{\wedge} \mathrm{T}_{\mathrm{e}}+\left(\delta \mathrm{K} / \delta \mathrm{M}_{\mathrm{e}}\right)^{\wedge} \mathbf{M}_{\mathrm{e}}
$$

Como en la dirección de $\lambda$ el gradiente se mantiene constante, entonces la función PIB sólo crece (o decrece) en las direcciones de $\mathrm{T}_{\mathrm{e}} \mathrm{y} \mathrm{M}_{\mathrm{e}}$, esto es, el comportamiento de la economía tiene una correlación directamente proporcional a la cantidad total de fuerza de trabajo esclava con que se cuenta, es decir, que se encuentra en la sociedad y la que es importada.

Este resultado implica que los cambios en el volumen de trabajo esclavo llevan consigo cambios en la economía y con ello es posible seguir el rastro al proceso económico siguiendo la pista de la cantidad de esclavos con que se contaba. Lo cual no quiere decir que la relación se dé necesariamente en la vía inversa, es decir, que a cambios en el PIB se sigan cambios en el volumen de esclavos, pues esto no ha sido demostrado aún.

\section{Comportamiento y correlación: Precio de esclavos}

La teoría económica más simple afirma que el comportamiento de los precios de una mercancía está determinado por dos fenómenos: fluctuaciones en la masa monetaria, o restricciones en la oferta (lo cual a su vez será explicado por Marx ${ }^{8}$ como un aumento en los costos de producción y por ende un mayor empleo de fuerza de trabajo). Esto quiere decir que si se deja como constante la masa monetaria, entonces, en términos esquemáticos, ocurriría que a un aumento en el volumen de la fuerza de trabajo le correspondería, o una caída en los precios de esclavos o una disminución en su oferta. De manera análoga, si la fuerza de trabajo disminuye, ello implica una elevación en el número de esclavos ofrecidos o un alza en sus precios.

\footnotetext{
${ }^{7}$ Enriqueta Vila Vilar, Hispanoamérica y el comercio de esclavos: Los asientos portugueses (Sevilla: Escuela de Estudios Hispano-americanos, 1977); Marisa Vega Franco, El tráfico de esclavos con América: Asientos de Grillo y Lomelín, 1663-1674 (Sevilla: Escuela de Estudios Hispano-americanos, 1984).

${ }^{8}$ Marx, Contribución a la crítica.
} 
Pero como es evidente, estas correspondencias sólo se pueden dar en mercados perfectos y en situaciones de equilibrio en el empleo. Todo lo cual no se dio en la Nueva Granada del siglo XVII. A lo más se podría decir que en contextos urbanos es esperable que la fuerza de trabajo de la gente de todos los colores tenga un comportamiento elástico.

La forma de establecer la correspondencia entre los precios de los esclavos y la economía no es a través de los mecanismos de sustitución de la mano de obra que hacen énfasis en situaciones de equilibrio. Aquí el camino para demostrar la correspondencia es a través de la observación del comportamiento de la generación de valor frente a cambios en la fuerza de trabajo incorporada al proceso de producción.

Para empezar se debe recordar que en las páginas anteriores se dijo que entre la fuerza de trabajo esclava y las otras formas de coerción existía una correspondencia, para el total de la economía, que se podía expresar a través de curvas de indiferencia, las cuales dependían del acceso a cada tipo de mano de obra y de los precios de éstas. Ahora lo que se hará es ver con algún detalle la correlación entre las tres variables: volumen, precio y producción.

En la ley de las proporciones variables de la fuerza de trabajo, se demuestra que la relación entre trabajo incorporado y producción total de la economía tiene tres intervalos diferentes. En el primero de ellos a cambios en la cantidad de trabajo se sigue una alza en la producción, la cual es marginalmente creciente. En el segundo intervalo, también es directamente proporcional el vinculo entre las dos variables, pero los rendimientos marginales son constantes. En el último intervalo, la relación es marginalmente decreciente.

Ahora bien, a cada uno de estos intervalos le corresponden curvas de indiferencia distintas, en tanto cada uno tiene niveles de producción diferentes, es decir, las parejas de fuerza de trabajo esclava y otras fuerzas de trabajo son combinaciones distintas en las cuales no se ha mantenido constante la utilidad producida por ella. Por esto se debe ver en detalle cada uno de los intervalos.

En el primero, como se ha dicho, se tienen relaciones marginalmente crecientes. Esto quiere decir que a pequeños cambios en la fuerza de trabajo se siguen cambios mayores en la producción. De lo cual se infiere que los precios de la mano de obra en su conjunto se elevan, en tanto a su introducción corresponden valores agregados más altos. Para el segundo intervalo, en el cual, se mantiene constante la relación, los precios de la mano de obra deben estabilizarse, en tanto la producción y la fuerza de trabajo tiene una 
relación que tiende hacía la linealidad. En el intervalo final, los precios tienden a la baja, pues se deben incorporar grandes volúmenes de mano de obra para conseguir rendimientos cada vez menores.

En pocas palabras, los precios de la mano de obra, cuando se va elevando la producción, registran tres etapas: crecimiento, estabilidad y descenso. Sin embargo, se puede pensar que en el caso de la economía colonial este comportamiento no ha sido así, pues existían mecanismos compulsivos y por ende las fluctuaciones no están tan fuertemente marcadas. Pero esto es cierto para las otras formas de trabajo diferente a la esclava (aunque probablemente también estén exentas las de los libres), pues para este caso los precios fluctúan de manera libre, pues los importados se vendían en subasta en Cartagena y los que ya existían se transaban sin ningún tipo de restricción frente al precio. Por lo tanto, si el precio de estos crecía es porque la producción total también lo hacía. De igual forma, si caían los precios, se puede inferir que la economía entraba en una recesión, no porque la esclavitud determine el movimiento de la economía, sino simplemente porque las series económicas de la esclavitud tienen un correlato en el comportamiento económico del Nuevo Reino.

Ahora bien, se podría pensar que los precios de los esclavos podrían tener un comportamiento deferente al de la economía, en tanto estos se podrían manipular por parte de los monopolios y oligopolios del comercio colonial, de tal forma que crecieran de manera artificial y no precisamente por las fluctuaciones propias del mercado. Sin embargo, esta hipótesis no es del todo convincente si se analiza a la luz de los tres fenómenos más influyentes en el mercado atlántico del siglo XVII, es decir, la tensión entre la Casa de Contratación y el Consulado de Sevilla; el sistema de importación de esclavos por parte de agentes externos a la corona española; y obviamente el comercio ilegal, impulsado tanto por las potencias no ibéricas como el generado por los movimientos entre las colonias americanas.

En un reciente artículo, Enriqueta Vila Vilar ${ }^{9}$ hablaba acerca de las tensiones y del papel fundamental del Consulado de Sevilla y en particular como éste monopolizó a lo largo del siglo XVII, el comercio entre España y sus colonias, ganándole el lugar a la Casa de Contratación. Pero a pesar de esto jamás fue posible para los comerciantes sevillanos el

\footnotetext{
${ }^{9}$ Enriqueta Vila Vilar, "El poder del consulado sevillano," en Relaciones de poder y comercio colonial: Nuevas perspectivas, ed. Enriqueta Vila Vilar y Allan J. Kuethe (Sevilla: Escuela de Estudios Hispanoamericanos, 1999).
} 
control del mercado de esclavos, a la vez que tampoco logró restringir el comercio por Buenos Aires y menos aún suprimir el comercio interregional en América. Estos tres factores aportaron para que el sueño de la Corona de un monopolio sobre el comercio de sus colonias fuera una simple ilusión.

Así, queda descartada la primera vía para argumentar un comercio monopólico de esclavos, en tanto los organismos de la Corona que soñaban con él jamás lograron establecerlo. Sin embargo se puede aún argumentar, que los agentes que conseguían los asientos si podrían controlar el mercado hasta tal punto que se pudiera hablar de un oligopolio de parte de ellos. Pero para matizar esta hipótesis nos debemos remitir a dos circunstancias particulares de este tráfico. ${ }^{10}$ Estas son: que los primeros agentes de contrabando de esclavos son los mismos asentistas, pues el sistema legal les permitía introducir un cierto número de piezas, pero ellos intentaban burlar este tope a través de innumerables artimañas y de esa forma elevar su ganancia.

Entre las formas de burlar el sistema las dos más características, son las arribadas forzosas y la simple corrupción de los funcionarios en los puertos autorizados para la trata. Esta segunda implicaba, que el comerciante al momento de llegar al puerto sobornaba a los agentes de la Corona de tal manera que ellos reportaban que el cargamento de esclavos se encontraba dentro de los límites contratados por la Corona, cuando en realidad estos lo superaban ampliamente. Por ejemplo, esta práctica supuso que en el caso de Buenos Aires se introdujeran entre 35,000 y 40,000 esclavos durante el período 1590 a 1640 cuando lo autorizado era de $2,000,{ }^{11}$ y el hecho es similar para Cartagena al ser el puerto más importante de la época y al que los traficantes preferían dirigirse, así sus permisos los obligaran a remitirse a Veracruz, en tanto en Tierra Firme era más común estos actos de corrupción. ${ }^{12}$

Ahora bien, esta práctica suponía que los esclavos no reportados oficialmente se vendían por fuera de los mecanismos oficiales, con lo cual hay una primera oportunidad de ruptura de los precios oligopólicos, pues es diferente el precio de un esclavo comprado con todos los derechos reales y otro que no lo fue. Pero el caso más importante para confrontar los precios y liberalizarlos es el del contrabando como tal, el cual implicaba

\footnotetext{
${ }^{10}$ Aquí sólo enunciaremos estos dos fenómenos en tanto una explicación sistemática de ellos desborda los objetivos de este artículo. Mayor información sobre los mecanismos de importación de esclavos se puede encontrar en los textos de Moutoukias, Vila Vilar y Vega Franco ya citados.

${ }^{11}$ Moutoukias, "Contrabando y sector externo," 177.

${ }^{12}$ Vila, Hispanoamérica, 167.
} 
una fuerte oferta de esclavos. Por ejemplo entre 1690 y 1723, las flotas legales dirigidas a Cartagena y Portobelo fueron solamente siete, mientras que en el mismo período entraron al Pacífico 148 embarcaciones francesas. ${ }^{13}$ Lo que demuestra que el tráfico ilegal podía superar, o al menos equiparar, el tráfico legal. Sin contar, como hemos dicho, que el comercio legal estaba impregnado de ilegalidad.

Por último habría que agregar el comercio intercolonial, del cual aún no sabemos lo suficiente, pero con toda seguridad también implicó un fuerte movimiento de esclavos al interior de las Colonias y es necesario que se realicen esfuerzos para cuantificar y dimensionar el tamaño de estos circuitos.

Por todo ello podemos afirmar, con cierto grado de confianza, que los precios de los esclavos no eran plenamente controlables por parte de los monopolios oficiales, en tanto los continuos casos de contrabando lograban contribuir a generar una oferta adicional de fuerza de trabajo, que no sólo suponía más hombres de los permitidos en los puertos oficiales, sino también esclavos incorporados por puertos alternos a través de las arribadas forzosas y de las rutas del contrabando.

\section{Conclusiones}

En conclusión, se ha afirmado en estas páginas que si se establecen las curvas de volúmenes y precios de esclavos se puede determinar, a través de ellas, el comportamiento de toda la economía, es decir, si el costo de los esclavos crece y su número también, entonces, la economía está creciendo. Por el contrario, si ambos caen se puede afirmar de manera legítima que la economía esta en una fase de contracción. Además, si los precios descienden y los volúmenes aumentan, es porque los esclavos están siendo dirigidos hacía nuevos sectores de la economía, en tanto hay una sobre oferta de ellos. Con lo cual es probable que el sistema en general este a punto de empezar un nuevo ciclo expansivo. Por último, si los precios se elevan pero las cantidades no lo hacen, entonces hay una carencia en el mercado y la economía tiene márgenes para crecer pero no cuenta con fuerza de trabajo para lograrlo, es decir, se encuentra en un nivel de estancamiento. Todo lo cual no significa, repetimos, que el comercio de esclavos determine el comportamiento económico, simplemente que ambas tendencias se comportan de manera paralela y que es posible leer la realidad económica a través de la representación emanada del movimiento de preciso y volúmenes de esclavos introducidos.

\footnotetext{
${ }^{13}$ Ibid.
} 